\title{
Iterative Fixed-Point Methods for Solving Nonlinear Problems: Dynamics and Applications
}

\author{
Juan R. Torregrosa, ${ }^{1}$ Alicia Cordero, ${ }^{1}$ Vinay Kanwar, ${ }^{2}$ and Jisheng Kou ${ }^{3}$ \\ ${ }^{1}$ Instituto de Matemáticas Multidisciplinar, Universitat Politècnica de València, 46022 Valencia, Spain \\ ${ }^{2}$ University Institute of Engineering and Technology, Panjab University, Chandigarh 160014, India \\ ${ }^{3}$ School of Mathematics and Statistics, Hubei Engineering University, Xiaogan, Hubei 432000, China
}

Correspondence should be addressed to Juan R. Torregrosa; jrtorre@mat.upv.es

Received 4 December 2014; Accepted 4 December 2014; Published 24 December 2014

Copyright (c) 2014 Juan R. Torregrosa et al. This is an open access article distributed under the Creative Commons Attribution License, which permits unrestricted use, distribution, and reproduction in any medium, provided the original work is properly cited.

The fixed-point operator plays a significant as well as remarkable role in the study of nonlinear phenomena occurring in engineering, physics, economics, life sciences, and medical sciences. The design of fixed-point iterative methods for solving nonlinear problems, in particular nonlinear equations or systems, has gained a spectacular development in the last two decades. Nevertheless, the existence of recent and extensive literature on these iterative schemes reveals that this topic is still a dynamic branch of the applied mathematics with interesting and promising applications.

In the recent years, the study of the dynamical behavior of the rational operator associated to an iterative method has also become a rapidly growing area of research, since the dynamical properties of the rational operator give us important information about the convergence, efficiency, and reliability of the iterative method.

This special issue was opened in April of 2014 and closed in September of 2014. There were 34 submissions in total and 13 of them were accepted for publication, after strict reviews, which gave important developments in iterative methods and their applications. The guest editors of this special issue hope that the presented results could outline new ideas for futures studies.

The purpose of this special issue was to explore the last advances in the field of fixed-point iterative methods for solving nonlinear problems and their applications in mathematics and applied sciences. The authors were invited to submit original research articles to stimulate the continuing efforts in nonlinear equations and related theories. The special issue provided a forum for researchers and scientists to communicate their recent developments and to present their novel results on nonlinear problems.

The topics of the accepted papers cover the area from theory to real applications. With the help of linear and nonlinear functional analysis tools and also helped by real and complex dynamical analysis, several methods and their corresponding convergence analysis have been presented for solving nonlinear problems. All of them gave numerous numerical tests and some of them real applications. We have the pleasure to present the selected manuscripts for this special issue.

R. Chugh and S. Kumar extended a previous result of D. Zhang et al. from two countable families to $k$ countable families and prove weak and strong convergence results of two new multistep iterative processes to common fixed point of countable family of multivalued quasi-nonexpansive mappings in a uniformly convex Banach space.

Two manuscripts are devoted to the analysis of iterative methods for solving nonlinear equations. In "New Mono- and Biaccelerator Iterative Methods with Memory for Nonlinear Equations," the authors construct new iterative schemes with memory for solving nonlinear scalar equations of orders twelve and fourteen, by using accelerating parameters and needing only four functional evaluations per step. On the other hand, B. Campos et al. analyze the $(\alpha, c)$-family of iterative procedures on quadratic polynomials under a dynamical point of view. The authors find some values of the parameters 
$\alpha$ and $c$ whose associated iterative schemes show stable and unstable behavior.

W. Zhou and J. Kou present a third-order variant of Potra-Pták's iterative method for solving nonlinear systems in Banach spaces, by using a new vector extrapolation technique, whose local and semilocal convergence are proved.

In their manuscript, M. J. Martínez et al. use the fixed point iterative scheme to correct the errors between Hipparcos and ICRF2 Celestial catalogues. This allows the authors to link both systems, giving a uniform reference regardless the celestial body (an astronomical radio source) is visible or not.

In the manuscript "Existence and Algorithm for the System of Hierarchical Variational Inclusion Problems," the authors study the existence and approximation of solution of this kind of problems in Hilbert spaces. By using Maingés approach, N. Wairojjana and P. Kumam improve and generalize some known results in this area.

In the context of iterative methods for solving nonlinear matrix equations, four papers have been presented. M. K. Razavi et al. show a new iterative method for computing the approximate inverse of a nonsingular matrix. Moreover, F. Soleymani et al. describe an iterative process with cubical rate of convergence for finding the principal matrix square root. Application of this approach is illustrated by solving a matrix differential equation. On the other hand, in "An Algorithm for Computing Geometric Mean of Two Hermitian Positive Definite Matrices via Matrix Sign," the authors present a fast algorithm for computing the geometric mean of two matrices of this specific type. Finally, in "Approximating the Matrix Sign Function Using a Novel Iterative Method," the authors present an iterative method for finding the sign of a square complex matrix.

L. Guo et al. use the reproducing kernel iterative method to solve a class of two-point boundary value problems. By homogenizing the boundary conditions, the two-point boundary value problems are converted into the equivalent nonlinear operator equation. They prove that both solutions are equivalent and show the analysis of error and the numerical stability of the method.

In "Existence of Solutions for a Coupled System of Second and Fourth Order Elliptic Equations," the author generalizes quasilinearization technique to obtain a monotone sequence of iterates converging uniformly and quadratically to a solution of this kind of systems.

Finally, in "The Hybrid Steepest Descent Method for Split Variational Inclusion and Constrained Convex Minimization Problems," the authors introduce new implicit and new explicit iterative schemes for finding a common element of the set of solutions of a constrain convex minimization problems and the set of solutions of the split variational inclusion problem.

\section{Acknowledgments}

The guest editors would like to express their gratitude to all researchers who submitted papers for publication and to the many reviewers whose reports were essential for us. We would also like to thank the editorial board members of this journal, for their support and help throughout the preparation of this special issue. Alicia Cordero and Juan R. Torregrosa were partially supported by Ministerio de Ciencia y Tecnología MTM2011-28636-C02-02.

Juan R. Torregrosa Alicia Cordero Vinay Kanwar Jisheng Kou 


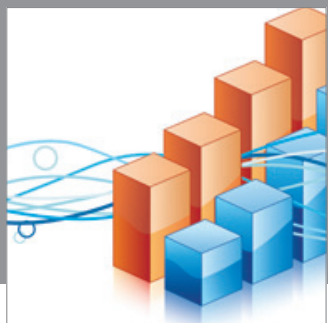

Advances in

Operations Research

mansans

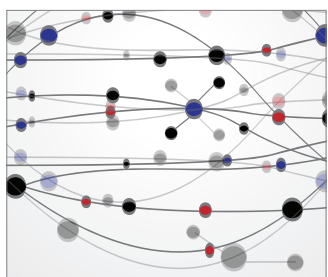

The Scientific World Journal
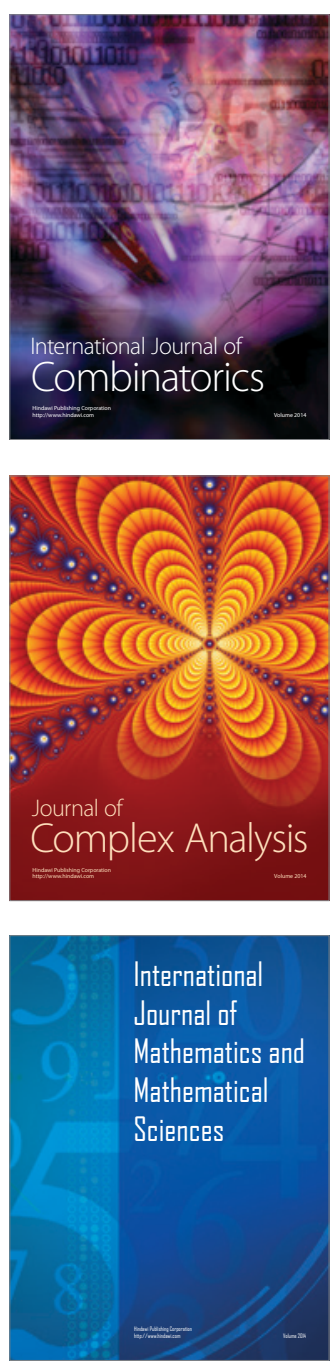
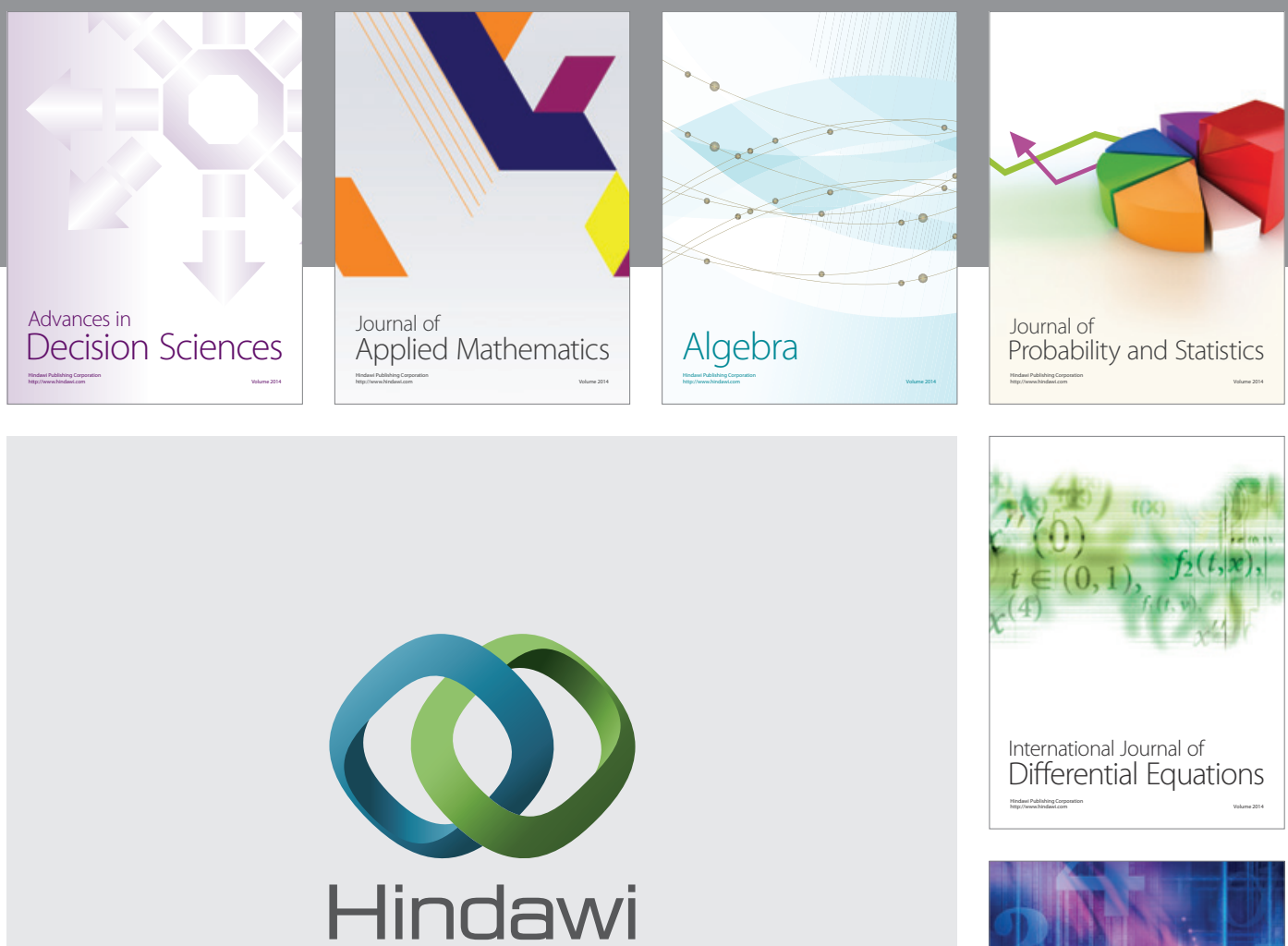

Submit your manuscripts at http://www.hindawi.com
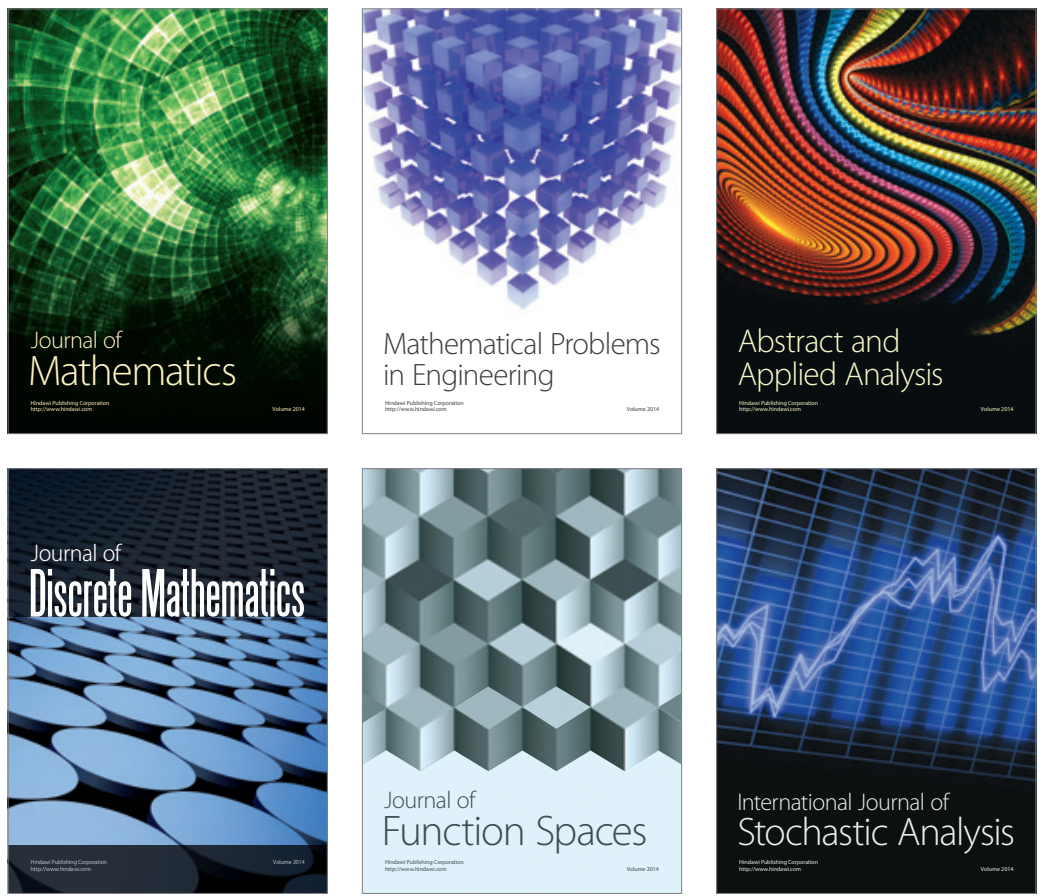

Journal of

Function Spaces

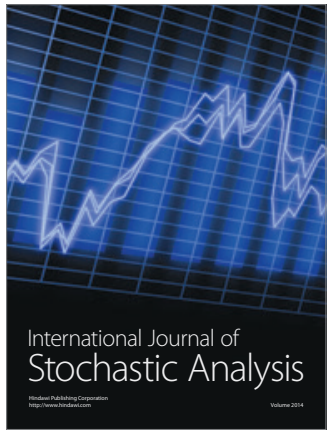

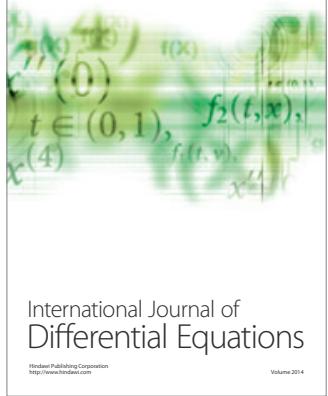
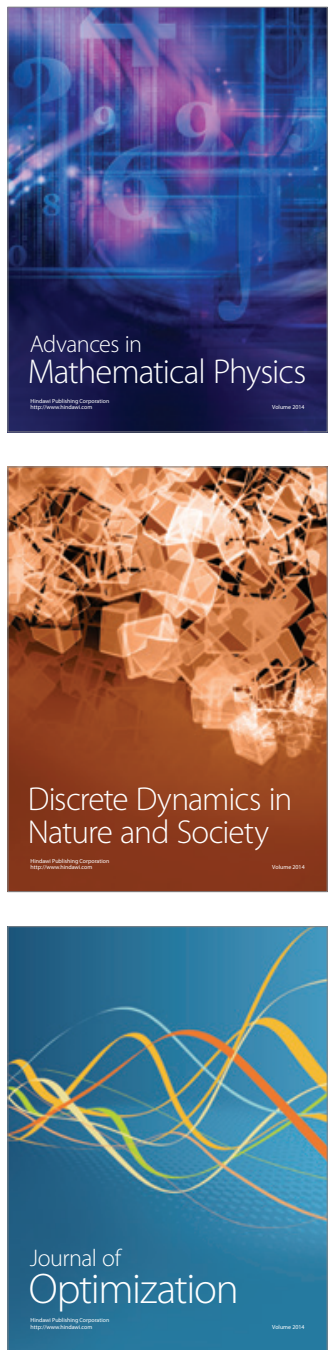